Draft Version SEPtember 27, 2018

Typeset using IATEX RNAAS style in AASTeX62

\title{
A Serendipitous Hard X-ray Detection of the Blazar LBQS 1319+0039
}

\author{
G. C. Privon, ${ }^{1}$ C. Ricci, ${ }^{2,3,4}$ F. E. Bauer, ${ }^{5,6,7}$ M. Á. Pérez-Torres,${ }^{8,9}$ R. Herrero-Illana, ${ }^{10}$ E. Treister, ${ }^{5}$ and S. Aalto ${ }^{11}$ \\ ${ }^{1}$ Department of Astronomy, University of Florida, PO Box 112055, Gainesville, FL 32611, USA \\ ${ }^{2}$ Núcleo de Astronomía de la Facultad de Ingeniería, Universidad Diego Portales, Av. Ejército Libertador 441, Santiago, Chile \\ ${ }^{3}$ Kavli Institute for Astronomy and Astrophysics, Peking University, Beijing 100871, China \\ ${ }^{4}$ Chinese Academy of Sciences South America Center for Astronomy, Camino El Observatorio 1515, Las Condes, Santiago, Chile \\ ${ }^{5}$ Instituto de Astrofsica, Facultad de Fsica, Pontificia Universidad Catlica de Chile, Casilla 306, Santiago 22, Chile \\ ${ }^{6}$ Space Science Institute, 4750 Walnut Street, Suite 205, Boulder, Colorado 80301 USA \\ ${ }^{7}$ Millenium Institute of Astrophysics, Santiago, Chile \\ ${ }^{8}$ Instituto de Astrofsica de Andaluca - CSIC, PO Box 3004, 18008, Granada, Spain \\ ${ }^{9}$ Visiting Scientist: Facultad de Ciencias, Univ. de Zaragoza, Spain \\ ${ }^{10}$ European Southern Observatory (ESO), Alonso de Córdova 3107, Vitacura, Casilla 19001, Santiago de Chile, Chile \\ ${ }^{11}$ Department of Space, Earth and Environment, Onsala Space Observatory, Chalmers University of Technology, 439 92, Onsala, Sweden
}

Keywords: galaxies: active — quasars: individual (LBQS 1319+0039) — X-rays: galaxies

We report a serendipitous hard X-ray (3-24 keV) detection of the blazar LBQS 1319+0039. This detection was obtained during a $20.7 \mathrm{ks}$ Nuclear Spectroscopic Telescope Array (NuSTAR; Harrison et al. 2013) observation of NGC 5104 (Program 03190, Sequence ID: 60370003002; G. C. Privon et al. in preparation). During the analysis of the observations, hard X-ray emission was identified at a position consistent with that of the blazar LBQS 1319+0039, which has cataloged coordinates of $13 \mathrm{~h} 21 \mathrm{~m} 39.5659 \mathrm{~s}+00 \mathrm{~d} 23 \mathrm{~m} 57.638 \mathrm{~s}$ (J2000; Petrov 2011) and a redshift $z=1.62$ (Hewett \& Wild 2010). LBQS 1319+0039 was classified as a flat-spectrum radio quasar by Massaro et al. (2009). For larger studies of NuSTAR serendipitous sources see Alexander et al. (2013) and Lansbury et al. (2017).

The data collected by NuSTAR were processed with the NuSTAR Data Analysis Software NUSTARDAS v1.8 within Heasoft v6.24, using the calibration files released on UT 2018 August 18, and following the same procedure reported in Ricci et al. (2017a). The spectra were binned to have one count per bin, and we applied Cash (1979) statistics to fit the data. We modeled the X-ray spectrum with a simple power-law model, considering also a constant to account for possible cross calibration between the two focal plane modules on board NuSTAR (CONST $\times$ ZPO in XSPEC).

The X-ray spectrum (Figure 1 ) is consistent $(\mathrm{C}$-stat $=194.6$ for 233 degrees of freedom) with powerlaw emission with a photon index of $\Gamma=1.72_{-0.32}^{+0.33}\left(F_{\nu} \propto \nu^{-\alpha}\right.$ where $\left.\Gamma=\alpha+1\right)$, while the cross-calibration constant is $C=0.93_{-0.22}^{+0.30}$. The observed emission and published redshift imply an observed $2-10 \mathrm{keV}$ luminosity of $\mathrm{L}_{2-10 \mathrm{keV}} \simeq 3.4 \times 10^{45} \mathrm{erg}$ $\mathrm{s}^{-1}$. The observed photon index is consistent with the median value found for Swift/BAT hard X-ray selected blazars $(\Gamma=1.68 \pm 0.04$, Ricci et al. 2017b, see also Ajello et al. 2009). For this source Massaro et al. (2009) report a $0.1-2.4$ $\mathrm{keV}$ flux corresponding to a luminosity of $2.4 \times 10^{45} \mathrm{erg} \mathrm{s}^{-1}$, which would translate into a luminosity of $2.4 \times 10^{45}$ in the $2-10 \mathrm{keV}$ range for $\Gamma=1.68$. We thus see no evidence for strong variability.

GCP acknowledges support from NuSTAR award 80NSSC17K0623 and the University of Florida. CR acknowledges the CONICYT+PAI Convocatoria Nacional subvencion a instalacion en la academia convocatoria año 2017 PAI77170080. ET acknowledges support from FONDECYT Regular 1160999, CONICYT PIA ACT172033 and BASAL CATA AFB 170002. 

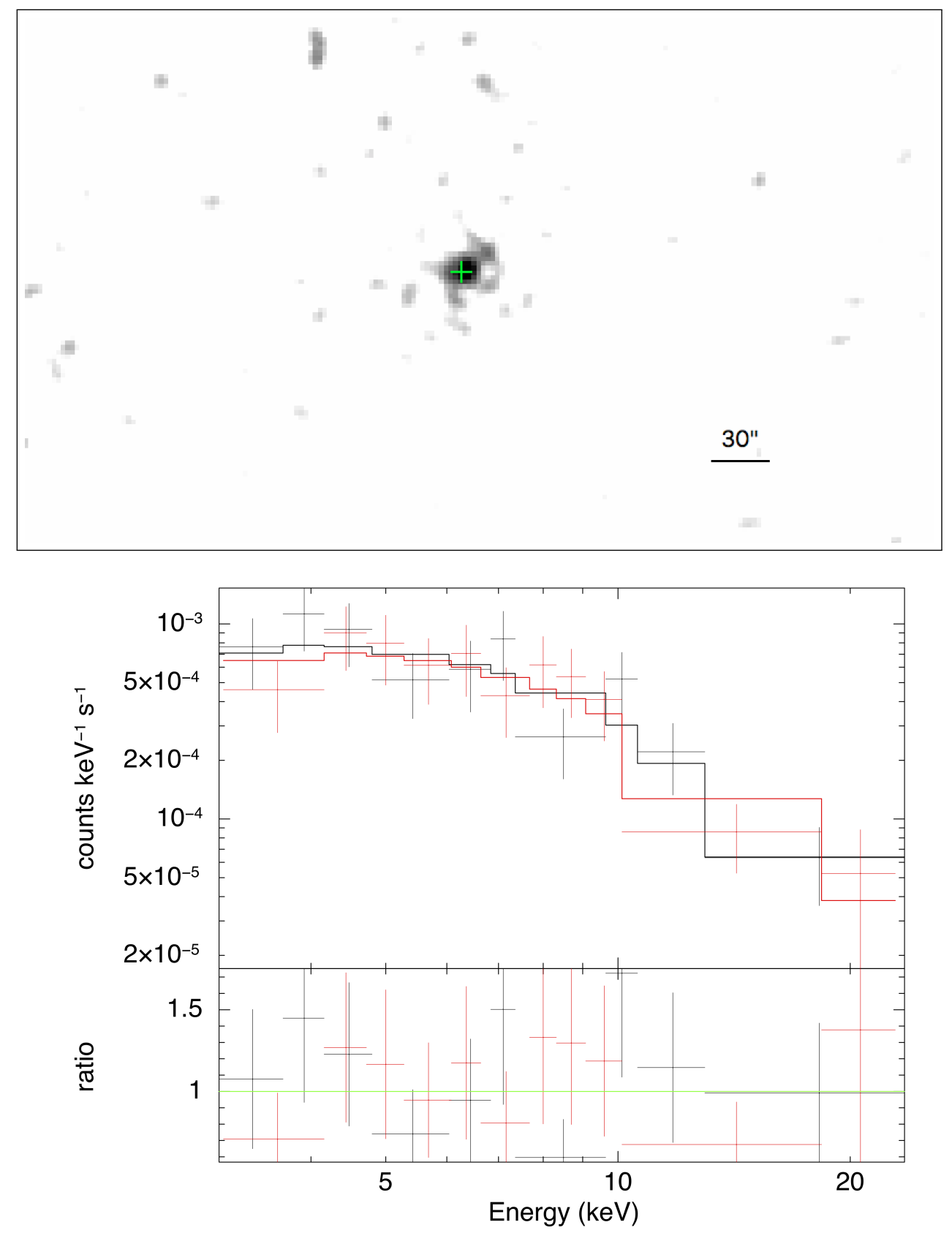

Figure 1. Top panel: $3-24 \mathrm{keV}$ image of the field around the detected serendipitous source. The green cross marks the position of the blazar LBQS 1319+0039 from Petrov (2011). Bottom panel: NuSTAR X-ray spectrum of LBQS 1319+0039. Black and red crosses denote the measurements from the two NuSTAR Focal Plane Modules A and B, respectively. The corresponding black and red lines show the best-fit models to the X-ray spectrum, which includes a cross-calibration constant and a power-law component.

\section{REFERENCES}

Ajello, M., Costamante, L., Sambruna, R. M., et al. 2009, ApJ, 699, 603

Alexander, D. M., Stern, D., Del Moro, A., et al. 2013, ApJ, 773, 125

Cash, W. 1979, ApJ, 228, 939
Harrison, F. et al. 2013, ApJ, 770, 103

Hewett, P. C., \& Wild, V. 2010, MNRAS, 405, 2302,

Lansbury, G. B., Stern, D., Aird, J., et al. 2017, ApJ, 836, 99 
Massaro, E., Giommi, P., Leto, C., et al. 2009, A\&A, 495, 691

Petrov, L. 2011, AJ, 142, 105
Ricci, C. et al. 2017, MNRAS, 468, 1273

Ricci, C. et al. 2017, ApJS, 233, 17 